\title{
On the Origin of the Intensity Deficit in Neutron Compton Scattering
}

\author{
G.F.Reiter \\ Physics Department, University of Houston, Houston, Texas, USA \\ P.M. Platzman \\ Bell Labs Lucent Technologies, Murray Hill, New Jersey, USA
}

\begin{abstract}
Neutron Compton Scattering measurements in a variety of materials have shown a relative deficit in the total signal from hydrogen compared to deuterium and heavier ions. We show here that a breakdown in the Born-Oppenheimer approximation in the final states of the scattering process leads to such a deficit, and may be responsible for the effect.
\end{abstract}

s PACS numbers, 61.12.Ex, 61.25.Em,82.30.Rs

Deep Inelastic Neutron Scattering experiments on a variety of systems have found the integrated intensity of the signal from hydrogen to be smaller than expected, relative to heavy ions such as oxygen, or even deuterium, [1] given the well known nuclear cross-sections for these materials. This deficit has also been observed in electron Compton scattering. [2] This has been attributed to previously unknown physics at the very short time scales of the measurements [3], quantum entanglement between the struck proton and the other protons of the material [4], inadequacies in the treatment of the data [5,6], and a failure of the usual van Hove scattering formula to describe the data [7]. We show here that the effect can be explained within the usual formalism by a breakdown of the Born Oppenheimer approximation in the final state of the scattering process, which contains a very rapidly moving proton with sufficient energy to mix the electronic states of the system. These corrections are present for any scattering experiment, but only become significant for high momentum transfer.

We begin with the usual expression for the scattering cross-section when the spin of the neutron is not observed, in the large q limit in which only incoherent scattering remains.

$$
S(\vec{q}, \omega)=\Sigma_{\vec{n}}|<0| e^{-i \vec{q} \cdot \vec{R}_{1}}\left|E_{\vec{n}}>\right|^{2} \delta\left(\omega-\frac{\left(E_{\vec{n}}-E_{0}\right)}{\hbar}\right)
$$

where the ground state is taken to be the Born Oppenheimer ground state,

$$
<\vec{r}, \vec{R} \mid 0>=\Phi_{0,0}(\vec{R}) \alpha_{0}(\vec{r}, \vec{R})
$$

$\vec{r}$ are the coordinates of the electrons and $\vec{R}$ the coordinates of the ions. The $\mid E_{\vec{n}}>$ are energy eigenstates of the complete system of electrons and ions. $\alpha_{0}(\vec{r}, \vec{R})$ is the solution of the electronic problem with the protons and other ions at the positions $\vec{R}$, and $\Phi_{0,0}(\vec{R})$ is the ground state for the heavy particles in the potential energy surface defined by $\mid \alpha_{0}>. \overrightarrow{R_{1}}$ is the component of $\vec{R}$ denoting the position of the struck particle, There are in addition, a complete set of excited electronic states, for the ions at the same positions, denoted by $\alpha_{i}(\vec{r}, \vec{R})$. It is evident from Eq. 1that

$$
\int S(\vec{q}, \omega) d \omega=1
$$

for any q. The total intensity of the incoherent scattering is proportional to this integral times the total scattering cross-section for the scattering particle. Our point of view is that if this appears not to be the case, then some intensity is not being accounted for in the experimental integration.

It is usually assumed at this point, that the energy eigenstates of the full Hamiltonian can be adequately represented by the ground state for the electronic system and the excited states for the ions in the Born Oppenheimer potential for that ground state. The energies $E_{\vec{n}}$ are the sum of the electronic energy, $E_{n}^{\prime}(\vec{R})$ and the energy of the heavy ions in the effective potential defined by this energy surface. However, we wish to consider the limit that the transferred momentum is large, so that the recoiling proton has sufficient energy to mix the electronic states. In short, we must go beyond the Born Oppenheimer approximation in expanding the state $e^{i \vec{q} \cdot \overrightarrow{R_{1}}} \mid 0>$ in energy eigenstates, and consider the full Hamiltonian for the system, which includes the kinetic energy of the struck proton. We will treat this kinetic energy as a perturbation, to the extent that it acts on the electronic wavefunctions, and first calculate the energy eigenstates to lowest order. We take the full Hamiltonian to be 


$$
H=H_{0}(\vec{r}, \vec{R})+\Sigma_{i} \frac{P_{i}^{2}}{2 M}
$$

The BO energy eigenstates , $\left|\Phi_{n, j}>\right| \alpha_{n}>$ satisfy

$$
H_{0}(\vec{r}, \vec{R}) \alpha_{n}(\vec{r}, \vec{R})=E_{n}^{\prime}(\vec{R}) \alpha_{n}(\vec{r}, \vec{R})
$$

and

$$
\left(\Sigma_{i} \frac{P_{i}^{2}}{2 M}+E_{n}^{\prime}(\vec{R})\right) \Phi_{n, j}(\vec{R})=E_{n, j} \Phi_{n, j}(\vec{R})
$$

In addition to the kinetic energies of the heavy particles, obtained from $\left(\Sigma_{i} \frac{P_{i}^{2}}{2 M} \mid \Phi>\right) \mid \alpha_{n}>$ the leading term in the perturbation when acting on a state of the form $\left.|\Phi>| \alpha_{n}\right\rangle$ is

$$
\Sigma_{i} \vec{P}_{i}\left|\Phi>\cdot \vec{P}_{i}\right| \alpha_{n}>/ M
$$

The action of the momentum of the heavy particle on the electronic states is, from Eq. 5

$$
<\alpha_{m}\left|\vec{P}_{i}\right| \alpha_{n}>=i \hbar<\alpha_{m}\left|\frac{\delta H_{0}}{\delta \vec{R}_{i}}\right| \alpha_{n}>/\left(E_{n}^{\prime}-E_{m}^{\prime}\right)
$$

where the matrix elements are taken over the electronic coordinates, and the positions of the heavy particles are the same for all the states. This perturbation mixes the original Born-Oppenheimer energy eigenstates. For all the heavy particles except the one struck by the neutron, the momentum operator acting on the wavefunction of the particle gives a small overall contribution, which is the basis for the usual Born-Oppenheimer approximation. However for the struck particle, particle 1 in Eq. 1, this operator gives a large term, of order $\hbar \vec{q}$, and cannot be neglected.

We have then that the energy eigenstates for the full Hamiltonian are given to first order by

$$
\left|E_{n, i}>=\right| \Phi_{n, i}>\left|\alpha_{n}>+\Sigma_{m, j}<\Phi_{m, j}\right|<\alpha_{m}\left|\frac{i \hbar \frac{\delta H_{0}}{\delta \vec{R}_{1}}}{\left(E_{n, i}-E_{m, j}\right)\left(E_{n}^{\prime}-E_{m}^{\prime}\right)}\right| \alpha_{n}>\cdot \frac{\vec{P}_{1}}{M}\left|\Phi_{n, i}>\right| \Phi_{m, j}>\mid \alpha_{m}>
$$

We will neglect for the moment the shift of the energies for these states, as it is not essential for this work. The important point, is that because of the presence of the fast proton, the excited states have a significant overlap with the ground state, and as a consequence, there is some amplitude for the scattered neutron to transfer some of its energy to the electronic system. When Eq. 9 is used in Eq. 1, we see that the only component of the excited states wave function that is significant is that which couples it to the ground state. The sum in Eq. 1 extends over the indices of the electronic states, and for each electronic state, one sums over the spectrum of eigenstates of the ionic system in that electronic state. We have then that there is a contribution to the sum in Eq. 1 from each of the the excited electronic states, $S_{n}(\vec{q}, \omega)$ of the form

$S_{n}(\vec{q}, \omega)=\Sigma_{i, j}\left|<\Phi_{0,0}\right| e^{-i \vec{q} \cdot \overrightarrow{R_{1}}}\left|\Phi_{0, j}><\Phi_{0, j}\right| \frac{\hbar}{M} \cdot<\alpha_{0}\left|\frac{\frac{\delta H_{0}}{\delta \vec{R}_{1}}}{\left(E_{n, i}-E_{0, j}\right)\left(E_{n}^{\prime}-E_{0}^{\prime}\right)}\right| \alpha_{n}>\cdot \vec{P}_{1}\left|\Phi_{n, i}>\right|^{2} \delta\left(\omega-\frac{\left(E_{n, i}-E_{0,0}\right)}{\hbar}\right)$

We are going to approximate the electronic matrix element in Eq. 10 by evaluating it at the equilibrium positions of the heavy particles. Since only ionic states for which the struck particle has a momentum in the vicinity of $\hbar q$ will have any significant overlap with the state $<\Phi_{0,0} \mid e^{-i \vec{q} \cdot \vec{R}_{1}}$, we can assume the $\left|\Phi_{0, j}\right\rangle$ that are important in the sum have this property. Then this must be true of the states $\left|\Phi_{n, i}\right\rangle$ as well. Furthermore, for the heavy particles which have not been struck, henceforth referred to as the bath, the wavefunctions and energies in both the ground and excited states are unaffected, initially, by the scattering event. Hence the change in the heavy particle energies in both the excited and ground electronic states is dominated by the kinetic energy of the struck particle, $\frac{(\hbar q)^{2}}{2 M}$. Thus we can replace $\left(E_{n, i}-E_{0, j}\right)$ in the denominator of Eq. 10 by $\left(E_{n}^{\prime}-E_{0}^{\prime}\right)$. The electronic matrix element is then independent of the heavy particle energy indices, and we can use any basis for the particle wavefunctions that we like. In particular, we choose a plane wave basis. This also allows us to evaluate the action of $\vec{P}_{1}$, which we will replace with $\hbar q$. This approximation neglects the momentum of the particles in the initial state compared to $\hbar q$ when evaluating the matrix elements. In the energy delta function, we will keep the full kinetic energy $\frac{(\hbar(\vec{p}+\vec{q}))^{2}}{2 M}$ in the 
final state, and replace $E_{0,0}$ by $E_{0}^{\prime}+\frac{p^{2}}{2 M}$. None of these approximations are essential for our main point, but serve to simplify the result. We find then that the excited electronic states contribute to $S(\vec{q}, \omega)$

$$
\Sigma_{n} S_{n}(\vec{q}, \omega)=\Sigma_{n}\left|\frac{\hbar^{2} \vec{q}}{M} \cdot<\alpha_{0}\right| \frac{\frac{\delta H_{0}}{\delta \vec{R}_{1}}}{\left(E_{n}^{\prime}-E_{0}^{\prime}\right)^{2}}\left|\alpha_{n}>\right|^{2} \int n(\vec{p}) \delta\left(\omega-\left(E_{n}^{\prime}-E_{0}^{\prime}\right) / \hbar-\frac{\hbar q^{2}}{2 M}-\frac{\vec{p} \cdot \vec{q}}{M}\right) d \vec{p}
$$

where $n(\vec{p})$ is the diagonal matric element of the one particle density matrix for particle 1 in the momentum representation. We see that these terms give the same functional form in $q$ and $\omega$ as the usual impulse approximation result but shifted to high energies by the difference in energies of the electronic states.

The total intensity in these terms is compensated for in the sum in Eq. 1 by the reduction in intensity of the scattering in which there is no change in the electronic wave function. The ground state of the electronic system, in the presence of the fast proton, is no longer $\left|\alpha_{0}\right\rangle$, but is given by Eq. 9. When used in the sum in Eq. 1, it must be normalized. The normalization of this state leads to a reduction of intensity of the primary signal, again with the same approximations, by an amount $\Delta I$

$$
\Delta I=\Sigma_{n}\left|\frac{\hbar^{2} \vec{q}}{M} \cdot<\alpha_{0}\right| \frac{\frac{\delta H_{0}}{\delta \vec{R}_{1}}}{\left(E_{n}^{\prime}-E_{0}^{\prime}\right)^{2}}\left|\alpha_{n}>\right|^{2}
$$

This just compensates for the intensity transferred to the excited electronic states. To the extent that one measures only the intensity in the primary peak, Eq. 12 gives the apparent deficit in the crossection of the struck particle. We see that this varies with $q^{2}$ and $1 / M^{2}$, as well as the particulars of the electronic system.

Although a detailed calculation is necessary to evaluate the magnitude of the deficit in the intensity of the main peak, we can get some idea of the size of the effect by introducing dimensionless variables. A characteristic length scale for molecular systems is the radius of the Bohr orbit, $a$. A characteristic energy scale is $\frac{e^{2}}{2 a}$. Since

$$
\frac{\delta H_{0}}{\delta \overrightarrow{R_{1}}}=\Sigma_{i} \frac{e^{2}}{\left|\overrightarrow{r_{i}}-\overrightarrow{R_{1}}\right|^{2}}
$$

where the sum in Eq.13 is over all the electrons in the system, we find that an estimate of the magnitude of the effect is given by

$$
\Delta I \approx\left(\frac{\frac{2 N_{e f f} \hbar^{2} q / a}{M}}{e^{2} / 2 a}\right)^{2}
$$

where $N_{\text {eff }}$ is the effective number of terms in the sum appearing in Eq. 13.

For a q of $50 \AA^{-1}$ and $N_{\text {eff }}$ of $3, \Delta I \approx .15$. Using a perhaps more realistic scale for water, which would be the energy gap of about $6 \mathrm{ev}$, we would have an increase by a factor of four. The result is also very sensitive to the value of Neff. It is clear that without evaluating the integrals, there is considerable uncertainty in the magnitude of the effect, but that nevertheless, it is potentially of the right order of magnitude to explain the deficits. However, the result above cannot explain two features of the phenomenology of the deficit as it is observed in a variety of systems. The deficit is not observed to vary with $q^{2}[3,9]$ in the Neutron Compton Scattering measurements on water. Although the results of the Electron Compton Scattering experiments in formvar [2] are consistent with a $q^{2}$ dependence of the deficit for the small range of q measured, the great bulk of the available data, over a much wider range in $\mathrm{q}$, is obtained using neutrons. The data on the deficit in $\mathrm{Nb}$ and Pd hydrides [10]does show a strong q dependence, which, however saturates at large q. The saturation value of $\Delta I$ is about .4, which is four times the value in the deuterated $\mathrm{Nb}$ hydride, as the theory above would predict, but the deuterated material shows no variation with $\mathrm{q}$.

Also, as it stands, there is no explanation for the dependence of the deficit on the relative concentration of deuterium and ordinary water. [1] Since the matrix elements and the density of electronic states that determine the amplitude of the effect are presumably nearly the same in $\mathrm{D}_{2} \mathrm{O}$ and $\mathrm{H}_{2} \mathrm{O}$, we would expect no concentration dependence from the results above. Both of these inadequacies point to the coupling of the electronic states in the presence of a fast proton being too strong to use the weak coupling perturbation theory presented so far. The effect of the higher order terms in the coupling may be accounted for by introducing linewidths for the excited states due to transfer of energy in the electronic system to the ions. These linewidths are strongly enhanced by the presence of the fast proton.

The time scale for the relaxation of the density correlation function, $\left(\frac{q \Delta p}{M}\right)^{-1}[8]$, where $\Delta p$ is the momentum distribution width, is about 1 femtosecond in water, for typical momentum transfers of $35 \AA^{-1}$. This corresponds to a momentum width for the protons of about $5 \AA^{-1}$ [11]. It is known that the relaxation time for the lowest excited electronic state in water to the so called solvated electron state, is about 150 femtoseconds, [12] considerably longer 
than the time scale we are interested in. However, that is when the protons are moving at velocities comparable to $\frac{\Delta p}{M}$ above. When one of these protons has momentum of $30-40 \AA^{-1}$ the relaxation rate could be a factor of fifty faster, since this rate has a component that varies as $q^{2}$. That rate is comparable to the typical time scale of the scattering, and the decay of the excited state can play a major role in determining the intensity deficit. Furthermore, it is known [13] that the excited electron wave function extends over 5-10 water molecules, and that the rate of transfer of energy to the protons or deuterons depends on a decoherence time scale that is longer by a factor of two in $\mathrm{D}_{2} \mathrm{O}$ than in $\mathrm{H}_{2} \mathrm{O}$, providing a mechanism for the concentration dependence of the deficit. Although we are not be able to compute the electronic properties needed to make this argument precise, it is plausible that a stong coupling theory that includes the decay of the excited states can account for the concentration dependence in water.

To go beyond lowest order perturbation theory, we will use a mode coupling approximation to include the decay of the excited states. The intermediate scattering function, $\mathrm{S}(\mathrm{q}, \mathrm{t})$, is

$$
S(\vec{q}, t)=<0\left|e^{-i \vec{q} \cdot \overrightarrow{R_{1}}} e^{i \vec{q} \cdot \overrightarrow{R_{1}(t)}}\right| 0>=<0\left|e^{-i \vec{q} \cdot \overrightarrow{R_{1}}} e^{\frac{i}{\hbar}\left(H_{0}+\Sigma \frac{P_{i}^{2}}{2 m}\right) t} e^{i \vec{q} \cdot \overrightarrow{R_{1}}} e^{-\frac{i}{\hbar}\left(H_{0}+\frac{\Sigma P_{i}{ }^{2}}{2 m}\right) t}\right| 0>
$$

Since

$$
\Sigma \frac{p_{i}^{2}}{2 M} \Phi_{0,0}(\vec{R})=\left(E_{0,0}-E_{0}^{\prime}(R)\right) \Phi_{0,0}(\vec{R})
$$

it follows that the action of the Hamiltonian on the ground state in Eq. 15 can be replaced by

$$
e^{-\frac{i}{\hbar} E_{0,0} t} \Phi_{0,0}(\vec{R})=e^{-\frac{i}{\hbar} E_{0}^{\prime}(R) t} e^{-\frac{i}{\hbar} \Sigma \frac{P_{i}^{2}}{2 M} t} \Phi_{0,0}(\vec{R})
$$

Having done this, the function $e^{-\frac{i}{\hbar} E_{0}^{\prime}(R) t}$ commutes with the density operator, $e^{i \vec{q} \cdot \overrightarrow{R_{1}}}$, and can be combined with the Hamiltonian acting on the final state. There is an additional term, arising from the commutator of the kinetic energy term in Eq. 15 with $E_{0}^{\prime}(R)$. This gives the effects of the forces acting on the heavy particles. This term has no direct effect on the impulse approximation limit, [8], $q \rightarrow \infty, t \rightarrow 0$, qt finite, as it is of order $q t^{2}$. It leads to traditional final state corrections of order $1 / \mathrm{q}$ that are included in the data analysis. Thus we can include the ground state electronic energy in the exponent in Eq. 15. The kinetic energy terms that do not involve the struck particle can also be approximated as commuting with the electronic energy, since this is the essence of the Born-Oppenheimer approximation. The noncommutative terms are responsible for the thermalization of the electronic states. These lead to the relaxation processes mentioned earlier, and occur on a much longer time scale than the one we are interested in. We will therefore neglect the commutator of the kinetic energy of the unstruck particles with the electronic Hamiltonian. We can then commute the kinetic energy for the unstruck particles through the density operator and eliminate it from the direct time evolution of the density operator.

Finally, we can approximate the operator $P_{1}^{2}$ by

$$
P_{1}^{2}=P_{1}^{1^{2}}+2 P_{1}^{1} \cdot P_{1}^{2}
$$

where the operator $P_{1}^{1}$ acts only on the proton wave function, and the operator $P_{1}^{2}$ acts only on the parameters $\vec{R}$ of the electronic wavefunction. In its action on the ground state, we will ignore the second term on the right in Eq. 18, which is, again, the Born Oppenheimer approximation.

We will also replace $P_{1}^{1}$ by $\hbar \vec{q}$ in the second term of Eq. 18, when this operator acts on final states for which the momentum is approximately $\hbar \vec{q}$. The full Hamiltonian acting on the final state is now the sum of two commuting terms. allowing the operator $P_{1}^{1^{2}}$ to act on $e^{i \vec{q} \cdot \vec{R}_{1}}$ we can then express the intermediate scattering function as

$$
S(\vec{q}, t)=<0\left|e^{-i \vec{q} \cdot \overrightarrow{R_{1}}} e^{\frac{i}{\hbar}\left(H_{0}-E_{0}^{\prime}+\frac{\hbar \vec{q} \cdot \overrightarrow{P_{1}^{2}}}{M}\right) t} e^{i \vec{q} \cdot\left(\overrightarrow{R_{1}}+\frac{\overrightarrow{P_{1}^{1}}}{M} t\right)}\right| 0>
$$

If we neglect the perturbation $\frac{\hbar \vec{q} \cdot \overrightarrow{P_{1}^{2}}}{M}$ in Eq. 19 we obtain immediately the impulse approximation result for $\mathrm{S}(\mathrm{q}, \mathrm{t})$, making clear again that it is the mixing of the Born-Oppenheimer eigenstates that is the source of the intensity deficit.

Eq. 19 can be expressed in more detail, making use of the identity $e^{\frac{i \hbar q^{2}}{2 m} t} e^{i \vec{q} \cdot \overrightarrow{R_{1}}} e^{i \vec{q} \cdot\left(\frac{\overrightarrow{P_{1}^{1}}}{M}\right) t}=e^{i \vec{q} \cdot\left(\overrightarrow{R_{1}}+\frac{P_{1}^{1}}{M}\right) t}$, as

$$
S(\vec{q}, t)=e^{\frac{i \hbar q^{2}}{2 M} t} \int d \vec{r} d \vec{R} \Phi_{0,0}^{*}(\vec{R}) \alpha_{0}^{*}(\vec{r}, \vec{R}) e^{\frac{i}{\hbar}\left(H_{0}-E_{0}^{\prime}(\vec{R})+\frac{\hbar \vec{q} \cdot \overrightarrow{P_{1}^{2}}}{M}\right) t} e^{i \frac{\vec{q} \cdot \overrightarrow{P_{1}^{1}}}{M} t} \alpha_{0}(\vec{r}, \vec{R}) \Phi_{0,0}(\vec{R})
$$

For each value of $\mathrm{R}$, we see that we now have a standard perturbation problem for the action of the Hamiltonian on the electronic wave function. 
It is clear from the above, that it is the evolution of the electronic state in the presence of the fast proton that must be calculated to obtain the deficit. We choose to do the perturbation calculation for the electronic propagator in the frequency domain rather than directly in the time domain. Using standard projection operator methods for the the resolvent operator for the electronic Hamiltonian we can show

$$
<\alpha_{0}\left|\left[z-H_{0}+E_{0}^{\prime}(\vec{R})+\frac{\hbar \vec{q} \cdot \overrightarrow{P_{1}^{2}}}{M}\right]^{-1}\right| \alpha_{0}>=\left[z-\Gamma_{0}(z)\right]^{-1}
$$

where

$$
\left.\Gamma_{0}(z)=\Sigma_{n, m}^{\prime}\left(\frac{\hbar^{2} \vec{q}}{M}\right)^{2}<\alpha_{0}\left|\frac{\frac{\delta H_{0}}{\delta \vec{R}_{1}}}{\left(E_{n}^{\prime}-E_{0}^{\prime}\right)}\right| \alpha_{n}><\alpha_{n}\left|\left[z-\left(H_{0}-E_{0}^{\prime}\right) / \hbar-\frac{\hbar \vec{q} . \vec{P}_{1}^{2}}{M}\right]^{-1}\right| \alpha_{m}><\alpha_{m} \mid \frac{\frac{\delta H_{0}}{\delta \vec{R}_{1}}}{\left(E_{m}^{\prime}-E_{0}^{\prime}\right)}\right]^{-1} \mid \alpha_{0}>
$$

The prime on the summation in Eq, 22 indicates the that the the ground state is omitted from both sums. Furthermore, the perturbation $\frac{\hbar \vec{q} \cdot \overrightarrow{P_{1}^{2}}}{M}$ operator should be understood as having no matrix elements with the ground state.

We will approximate the sum in Eq. 22 by keeping only the diagonal terms. These are the leading terms in the perturbation series in powers of $q$. In this case, we have

$$
\Gamma_{0}(z)=\Sigma_{n}^{\prime} \frac{\left|\frac{\hbar^{2} \vec{q}}{M} .<\alpha_{0}\right| \frac{\frac{\delta H_{0}}{\delta \vec{R}_{1}}}{\left(E_{n}^{\prime}-E_{0}^{\prime}\right)}\left|\alpha_{n}>\right|^{2}}{z-\left(E_{n}^{\prime}-E_{0}^{\prime}\right) / \hbar-\Gamma_{n}(z)}
$$

where

$$
\Gamma_{n}(z)=\Sigma_{m}^{\prime} \frac{\left|\frac{\hbar^{2} \vec{q}}{M} \cdot<\alpha_{n}\right| \frac{\frac{\delta H_{0}}{\delta R_{1}}}{\left(E_{m}^{\prime}-E_{n}^{\prime}\right)}\left|\alpha_{m}>\right|^{2}}{z-\left(E_{m}^{\prime}-E_{0}^{\prime}\right) / \hbar-\Gamma_{m}(z)}
$$

The prime on the sum in Eq. 23 indicates that the ground state is omitted, and in Eq. 24 that both the ground state and the state $\mathrm{m}=\mathrm{n}$ are omitted. Eqs. 23,24 are an approximation, which should be understood as self-consistently defining the self energies. We will not attempt to calculate these. For our purposes, it is sufficient to note that these self energies are proportional to $q^{2}$ for small $q$, and can become independent of $q$ for large $q$.

The energies in Eq.23 depend on the position of the proton, which is to be averaged over in Eq. 20. The effect of this averaging is to produce a distribution of energies in the resolvent expression Eq. 23 that is independent of q and will therefore be neglected in comparison with the self energy of the excited states. However, while formally smaller in powers of $\mathrm{q}$, the electronic energies are large compared to $\frac{\hbar q \Delta p}{M}$, and their variation can introduce a time scale comparable to the decay time of $S(\vec{q}, t)$ for moderate values of q. In the work of Schwartz et al [13], it is shown that the decoherence factor $<e^{\frac{i}{\hbar}\left(E_{n}^{\prime}(\vec{R})-E_{0}^{\prime}(\vec{R})\right) t}>$ that arises from this averaging has a decay time of about 5 femtoseconds for deuterium, and about half that for protons. [13] Therefore the numerical value for $\Gamma_{0}(z)$ can be strongly influenced by this averaging, which must also include, in a realistic calculation, the average over spatial configurations of surrounding molecules. We believe this difference in decoherence time is responsible for the concentration dependence of the deficit in $\mathrm{H}_{2} 0-D_{2} \mathrm{O}$ mixtures. The shorter decoherence time produces smaller relaxation rates, smaller values for $\Gamma_{0}(z)$ and hence larger deficits. We neglect it here for simplicity only, in order to focus on the main point, the qualitative behavior of the intensity deficit with q, and evaluate the energies at the equilibrium position of the proton. The electronic propagator(resolvent) then becomes independent of $\overrightarrow{R_{1}}$ and the momentum of the proton is conserved by the dynamics. We find then, that the Laplace Transform of Eq. 19 is

$$
\int_{0}^{\infty} d t e^{-i z t} S(\vec{q}, t) d t=-i \int d \vec{p} n(p)\left[z-\frac{\hbar q^{2}}{2 M}-\frac{\vec{p} \cdot \vec{q}}{M}-\Sigma_{n} \frac{\left|\frac{\hbar^{2} \vec{q}}{M} \cdot<\alpha_{0}\right| \frac{\frac{\delta H_{0}}{\delta \vec{R}_{1}}}{\left(E_{n}^{\prime}-E_{0}^{\prime}\right)}\left|\alpha_{n}>\right|^{2}}{z-\left(E_{n}^{\prime}-E_{0}^{\prime}\right) / \hbar-\frac{\hbar q^{2}}{2 M}-\frac{\vec{p} \cdot \vec{q}}{M}-\Gamma_{n}\left(z-\frac{\hbar q^{2}}{2 M}-\frac{\vec{p} \cdot \vec{q}}{M}\right)}\right]^{-1}
$$

We can recover the results of the previous weak coupling analysis by ignoring $\Gamma_{n}(z)$ in the denominator of the expression in the sum in Eq. 25. The neutron scattering function, $S(\vec{q}, \omega)$ in Eq. 1 is obtained by letting $\mathrm{z} \rightarrow \omega-i \epsilon$ in Eq. 25 and taking the real part of the resultant expression. The amplitude of the pole at $\omega=\frac{\hbar q^{2}}{2 M}+\frac{\vec{p} \cdot \vec{q}}{M}$ is $1 /\left(1-\left.\frac{\delta \Gamma_{0}(z)}{\delta z}\right|_{z=\omega}\right)$ where $\Gamma_{0}(z)$ is the sum in Eq.25. This is the same result as Eq.12, to lowest order in q. With the inclusion of linewidths for the excited states, and hence lineshifts as well, we have the possibility of this intensity deficit saturating as $\mathrm{q} \rightarrow \infty$. What is required is that the lineshifts become comparable to the energy differences from the 
ground state for the excited states for which the matrix elements in the numerator are significant. While we cannot show that this occurs for the values of $q$ in the experiments, since we cannot evaluate the electronic matrix elements, we can show that in the limit of very strong coupling, in which the perturbation matrix elements are comparable or greater than the energy level separations, such a result holds. In Eq.20, we can write

$$
e^{\frac{i}{\hbar}\left(H_{0}-E_{0}^{\prime}(\vec{R})+\frac{\hbar \vec{q} \cdot \vec{p}_{1}^{2}}{M}\right) t} \approx e^{\frac{i}{\hbar}\left(H_{0}-E_{0}^{\prime}(\vec{R})\right) t} e^{\frac{i \vec{q} \cdot P_{1}^{2} t}{M}} e^{\frac{i}{2} \frac{\vec{q}}{M} \cdot \frac{\delta H_{0}}{\delta \vec{R}_{i}} t^{2}}
$$

if we neglect higher order terms in the Baker-Campbell-Hausdorf formula. The last term in Eq. 26 is of order $q t^{2}$. While this is formally of higher order than the terms kept in the impulse approximation limit, the coefficient is determined by the forces on the proton in the excited states, and is on the scale of electronic energies. For times of the order of the characteristic times that are actually achieved in the experiments, the last phase factor in Eq. 28 is approximately

$$
\frac{\frac{e^{2}}{2 a^{2}}\left(\frac{q \Delta p}{M}\right)^{-1}}{\Delta p} \approx 9
$$

and cannot be neglected. It has been included in the discussion above. It is nevertheless useful to consider the very large $\mathrm{q}$ limit, where this phase factor can be neglected. We then find that $\mathrm{S}(\mathrm{q}, \mathrm{t})$ can be written simply as

$$
S(\vec{q}, t) \approx e^{\frac{i \hbar q^{2}}{2 M} t} \int d \vec{R} \Phi_{0}^{*}(\vec{R}) \Phi_{0}\left(\vec{R}+\frac{\hbar \overrightarrow{q_{1}} t}{M}\right)<\alpha_{0}(\vec{R}) \mid \alpha_{0}\left(\vec{R}+\frac{\hbar \overrightarrow{q_{1}} t}{M}\right)>
$$

The notation $\overrightarrow{q_{1}}$ in Eq. 28 indicates that it is a vector corresponding to a displacement of the coordinate of particle 1 . It should be noted that the electronic matrix element is independent of $\vec{R}$ at $\mathrm{t}=0$, and will be weakly dependent on $\vec{R}$ for subsequent times, while the nuclear wavefunction depends strongly on $\overrightarrow{R_{1}}$ Ignoring the position dependence of the electronic overlap, we can then write the nuclear overlap integral in the momentum representation, and obtain the usual impulse approximation result for $S(\vec{q}, t)$ multipled by a time dependent factor. Our point here is that this factor approaches a constant in the large q limit for times of the order of the characteristic time. Since the time for which $S(\vec{q}, t)$ is significant is $\left(\frac{q \Delta p}{M}\right)^{-1}$, the electronic overlap integral, which is responsible for the intensity deficit, involves the two states separated by a finite amount, $\frac{\hbar}{\Delta p}$. Or using the uncertainty relation, by a separation comparable to the localization distance of the proton in the ground state. The reduction in intensity, therefore, is independent of $\mathrm{q}$ in the very strong coupling limit, and proportional to $q^{2}$ in the weak coupling limit. While we are not in the very strong coupling limit, we think it plausible that the experiments are being done in an intermediate region, with the perturbation comparable to energy level differences, and showing a weak q dependence. This could lead to a variety of dependences of the deficit on the electronic properties, as observed. While most experiments show an increase in the deficit with q, for instance, recent experiments on LaH2 and LaH3 show a slight decrease with increasing q at large q. [14]

If the intensity is transferred, it is important to know why it has not so far been observed. In water, the minimum excitation energy is about $6 \mathrm{ev}$, so that the intensity that is shifted by exciting the electronic system would be on the high energy side of the main signal by at least that amount. It would continue to higher energies by an amount that depended upon the sum of the widths of the occupied and unoccupied levels that were involved in the transitions. Using recent calculations of Cabral do Couto et al [15], we find this to be on the order of 30ev if the dominant contribution to the sums in Eq.23 come from the valence and conduction band, but the widths could be much larger if there is significant excitation of higher and/or deeper bands. It is difficult to say where the center of gravity of the shifted spectral density would lie, but it is easily conceivable that it lies at energies that correspond to transit times at Vesuvio of less than 50 milliseconds, which is where the data for water stops. It is also the case, that because of the collapse of the phase space for short times, where a large range of momentum transfers are incorporated in a small change in the time of flight, the signal may be missed due to detector saturation. The data for LaH2 and LaH3 is more problematical, as there is a band gap of about .5 ev in LaH3, while LaH2 is a metal [16] and yet there is very little difference in the magnitude of the intensity deficit. This could be explained by the center of gravity of the absorbtion being at energies much higher than the gap.

It is evident that to give a quantitative explanation for the intensity deficit in various materials, it is necessary to be able to calculate the integrals over the electronic levels with some accuracy. We have not done this, and indeed, it is possible that the effects we are calculating may be too small to explain the observations. What we have shown is that a straightforward physical effect, the mixing of Born-Oppenheimer levels and an increase in the linewidth of excited electronic levels when a proton of large momentum is present, effects that must be operative at some level in any case, have the capacity to account for the phenomenology of the intensity deficits in Neutron Compton Scattering, and we have provided a framework in which to accurately calculate these effects. 


\section{ACKNOWLEDGMENTS}

We would like to thank Eric Bittner and Sylvio Canuto for several useful conversations and, P. Cabral do Couto, Jerry Mayers and Tyno Abdul-Redah for making available work prior to publication. The authors are supported by DOE Grant 1-5-555229

[1] C. A. Chatzidimitriou-Dreismann, T. Abdul-Redah, F. Streffer, J. Mayers, Phys. Rev. Letts. 79, 2839,(1997);

[2] C. A. Chatzidimitriou-Dreismann, M. Vos, C. Kleiner, T. Abdul-Redah, Phys. Rev. Letts. 91, 057403,(2003);

[3] C. A. Chatzidimitriou-Dreismann, T. Abdul-Redah,F. Streffer, J. Mayers J. Chem. Phys. 116, 1511,(2002);

[4] E. B. Karlsson and S. W. Lovesey, Phys. Rev. A 61, 062714,(2000);E.B. Karlsson, Phys. Rev. Letts. 90, 095301,(2003)

[5] J.J. Blostein, J. Dawidovski and J.R. Granada, Physica B 304, 357, (2001)

[6] R. Cowley, J. Cond. Matt. Phys. 15, 4143, (2003)

[7] N. Gidopoulis, (preprint)

[8] R. Silver and G.reiter, Phys.Rev.Letts.54,1047,(1985)

[9] T. Abdul-Redah and J. Mayers, "Neutron Inelastic Cross-Sections at eV Energy Transfer", (submitted to J. Cond. Matt. Phys. )

[10] E. Karlsson et al, Phys. Rev. B, 67, 184108, (2003)

[11] G. Reiter, J.C. Li, J. Mayers, T. Abdul-Redah and P.Platzman, submitted to PRL

[12] K.F. Wong and P.Rossky, J. Chem. Phys. 116,8418, (2002)

[13] B. J. Schwartz, E. R. Bittner, O. V. Prezhdo and P. Rossky, J. Chem. Phys. 104, 5942, (1996)

[14] T. Abdul-Redah, private communication

[15] P. Cabral do Couto, R.C. Guedes, and B.J. Costa Cabral, Braz. J. Phys 34, 42 (2004)

[16] M. Gupta and J. P. Burger, Phys. Rev. B 22, 6074, (1980) 